\title{
Positron emission tomography for cardiac amyloidosis: Timing matters!
}

\author{
Vasvi Singh, $M D{ }^{a}$ and Sharmila Dorbala, $M D, M P H^{\mathrm{a}, \mathrm{b}}$ \\ a Division of Nuclear Medicine and Molecular Imaging, Department of Radiology, Brigham and \\ Women's Hospital and Harvard Medical School, Boston, MA \\ b Cardiac Amyloidosis Program, Brigham and Women's Hospital and Harvard Medical School, \\ Boston, MA
}

Received Dec 30, 2020; accepted Dec 30, 2020

doi: $10.1007 / \mathrm{s} 12350-021-02524-\mathrm{x}$

\section{See related article, pp. $779-789$}

The diagnosis of cardiac amyloidosis is often delayed due to a myriad of clinical presentations, nonspecific elevation of serum cardiac biomarkers, and prevailing knowledge deficit about the disease. Recent advances in multimodality cardiovascular imaging including transthoracic echocardiography-derived speckle tracking imaging, cardiac magnetic resonance (CMR), and radionuclide imaging techniques have led to a paradigm shift in the non-invasive diagnosis of cardiac amyloidosis. ${ }^{1,2}$ An apical sparing pattern of abnormal longitudinal strain on speckle tracking echocardiography has been demonstrated to enhance the diagnostic accuracy for cardiac amyloidosis, ${ }^{3}$ though in patients with chronic kidney disease, a greater number of false-positive apical sparing patterns may reduce the accuracy for this technique. ${ }^{4}$ Moreover, echo and CMR predominantly provide structural and functional data, and a distinction between transthyretin amyloid cardiomyopathy (ATTR-CM) and light chain amyloid cardiomyopathy (AL-CM) is not achievable. Such distinction in a timely manner is crucial to guide treatment with recently developed targeted drugs for ATTR-CM, ${ }^{5}$ or chemotherapy-based regimens or stem cell transplant for AL-CM. ${ }^{6}$

Reprint requests: Vasvi Singh, MD, Division of Nuclear Medicine and Molecular Imaging, Department of Radiology, Brigham and Women's Hospital and Harvard Medical School, 75 Francis St, Boston, MA 02115; vsingh7@bwh.harvard.edu

J Nucl Cardiol 2022;29:790-7.

$1071-3581 / \$ 34.00$

Copyright (c) 2021 American Society of Nuclear Cardiology.
Cardiac scintigraphy with bone avid radiotracers pyrophosphate (PYP), 3,3-diphosphono-1,2-propanedicarboxylic acid (DPD), and hydroxymethylene diphosphonate (HMDP) labeled with ${ }^{99 \mathrm{~m}_{\text {technetium }}}$ $\left({ }^{99 \mathrm{~m}} \mathrm{Tc}\right)$ has been shown to have high specificity for the non-invasive diagnosis of ATTR-CM, in the absence of a monoclonal gammopathy. ${ }^{7}$ Although this approach has been validated in individuals with typical structural changes of ATTR-CM and heart failure, the sensitivity of this technique for detection of early ATTR-CM is not known. Early diagnosis of ATTR-CM is now more imperative than ever before. A recently developed transthyretin stabilizer, tafamidis, ${ }^{5}$ that targets the precursor protein has significantly improved survival. However, it is expensive ( $>\$ 200,000 /$ year) and is associated with non-response (no response or death by 30 months) in nearly $30 \%$ patients. One of the key proposed reasons for a lack of response to tafamidis therapy is initiation of treatment in patients with late stage ATTR-CM with advanced heart failure ("point of no return"). Therefore, an imaging modality that is able to diagnose this disease early allowing patients to gain maximum benefit from cutting-edge therapies is warranted. Furthermore, at present there are very limited data on the usefulness of serial SPECT or CMR/ echocardiography imaging for assessment of disease progression or response to therapy. ${ }^{8}$

Targeted amyloid-binding radiotracers for positron emission tomography (PET), ${ }^{18} \mathrm{~F}$-florbetapir, flutemetamol, -florbetaben, and ${ }^{11} \mathrm{C}$-Pittsburgh Compound-B $\left({ }^{11} \mathrm{C}-\mathrm{PIB}\right)$, were originally developed for $\beta$ amyloid imaging in Alzheimer's disease. The structure of the PET amyloid tracers is fundamentally similar to thioflavin- $\mathrm{T}$, and they likely bind to the beta-pleated motif of amyloid fibril which explains their ability to detect all amyloid deposits, independent of the precursor protein. ${ }^{1}$ An added advantage of the ${ }^{18} \mathrm{~F}$-labeled 
radiotracers is a long half-life of 109.7 minutes, making it feasible for unit dose delivery to sites without a cyclotron and thereby increasing their availability. Notably, amyloid PET tracers are the only clinically accessible radiotracers that effectively image AL-CM. ${ }^{18}$ F-florbetapir, -flutemetamol, -florbetaben, and ${ }^{11} \mathrm{C}$ Pittsburgh Compound-B ( $\left.{ }^{11} \mathrm{C}-\mathrm{PIB}\right)$ have been demonstrated to successfully image AL-CM and ATTR-CM (Table 1). Both static semiquantitative metrics (targetto-blood pool ratio) and quantitative metrics (standard uptake value, SUV, or cardiac retention index, RI) of amyloid tracer PET imaging have demonstrated value for the diagnosis of cardiac amyloidosis (Table 1). Quantitative PET may prove to be extremely valuable for the non-invasive diagnosis of early cardiac amyloidosis and detection of change in amyloid burden with serial imaging. ${ }^{1,9}$ Indeed, emerging data suggest that ${ }^{18} \mathrm{~F}$-florbetapir ${ }^{10}$ and ${ }^{11} \mathrm{C}-\mathrm{PiB}{ }^{11}$ PET can diagnose early AL-CM, prior to increased LV wall thickening or cardiac biomarker release.

In this issue of the journal, Papathanasiou et al. ${ }^{12}$ sought to investigate the accuracy of ${ }^{18} \mathrm{~F}$-flutemetamol for the diagnosis of cardiac amyloidosis in 17 subjects (ATTR-CA $=10$, AL-CA = 2, non-amyloid heart failure controls = 5), using PET/MRI (13 subjects) and PET/CT (4 subjects). A dose of $5 \pm 0.5 \mathrm{mCi}$ of ${ }^{18} \mathrm{~F}$-flumetamol was used. Dynamic PET imaging starting with the injection of ${ }^{18}$ F-flutemetamol was performed in 5 subjects for 30 minutes. The remaining 12 subjects were imaged for a mean of 78 minutes post injection (all except one subject were imaged starting $>59$ minutes post injection), for one cardiac bed position of unclear duration. Only two of the 12 amyloidosis subjects (17\%) showed visually increased myocardial tracer uptake. These subjects showed a calculated retention index of 0.072 and 0.067 (similar ranges described in the literature for other tracers). Although SUVmax, SUVmean, TBRmax and TBRmean values trended higher in subjects with cardiac amyloidosis, they did not differ statistically from controls.

Table 1 summarizes the emerging body of literature from several different centers on imaging of cardiac amyloidosis using PET tracers. Most initial studies included small sample sizes and included subjects with AL-CM and ATTR-CM. But, as shown in that table, the collective multicenter experience of amyloid tracer PET imaging, and specifically with ${ }^{18} \mathrm{~F}$-flutemetamol, and in ATTR-CM, is limited and the findings of this single center study are important as they add to this emerging knowledge.

The authors discuss that this is the first study to demonstrate a low sensitivity for an amyloid-binding PET tracer to detect amyloid in the heart. This provocative idea, if valid, may have important clinical implications. We believe that three key factors-patient characteristics, protocol factors, tracer characteristicsmay explain the reported low myocardial ${ }^{18} \mathrm{~F}$ flutemetamol activity reported in this study.

First, there is heterogeneity of patient cohort and imaging protocols. Of the 12 subjects with cardiac amyloidosis, only 2 had AL-CM, and these results are thus not applicable to AL-CM. The small $(N=10)$ ATTR cohort size limits meaningful reporting of test performance metrics such as test sensitivity. Even within this small group, there was heterogeneity in post-injection image timing (and variation in scanner use, PET/CT and PET/MR).

Next, this study predominantly included ATTR-CM subjects. Prior patient studies of amyloid PET tracers, ${ }^{13}$ and an autoradiography study with ${ }^{18} \mathrm{~F}$-florbetapir, ${ }^{14}$ have shown that compared to AL-CM, myocardial signal is low in ATTR-CM. The dose of ${ }^{18} \mathrm{~F}$-flutemetamol was only $5 \mathrm{mCi}$ (lower than reported with other PET amyloid tracers), the duration of one bed position cardiac imaging ${ }^{11,15}$ is not reported, and images were acquired late. Also, imaging was performed later after injection of ${ }^{18} \mathrm{~F}$-flutemetamol. In contrast to prior studies where myocardial uptake was reported in cardiac amyloidosis in images obtained $<30$ minutes post injection of the PET tracer (Table 1), in the current study all images except in 1 subject were obtained $\geq 59$ minutes post injection. ${ }^{12}$ Several studies using ${ }^{18}$ F-florbetaben, ${ }^{15}$ ${ }^{18}$ F-flutemetamol, ${ }^{16}$ and ${ }^{18}$ F-florbetapir, ${ }^{13}$ have demonstrated that myocardial signal is low in patients with ATTR-CM and non-amyloid controls specifically on the images obtained more than 30 minutes after injection of tracer. Indeed, a study of published myocardial and blood pool time activity curves reveals that the maximal separation of myocardial amyloid PET tracer activity between control and ATTR-CM subjects is between 5 to 15 minutes post injection. ${ }^{11,13,15}$ Prior studies also showed that myocardial PET tracer signal is low in ATTR-CA compared to AL-CA. The significantly delayed post-injection acquisition of images with a low radiotracer dose, and in a study cohort of predominantly ATTR-CM patients are the likely reasons for the low myocardial signal of ${ }^{18} \mathrm{~F}$-flutemetamol in this study.

Finally, tracer-related differences may explain the discordant findings of this study compared to prior studies. Only systematic studies of ${ }^{18} \mathrm{~F}$-flutemetamol PET with imaging at earlier time points and in larger cohorts of participants with ATTR-CM and AL-CM can clarify that point.

Despite these caveats, this cardiac amyloidosis study, with results contrary to prior publications on amyloid-binding PET tracer imaging, is thought provoking. The results of this study support data from prior literature and suggest that future studies of ${ }^{18} \mathrm{~F}$ - 


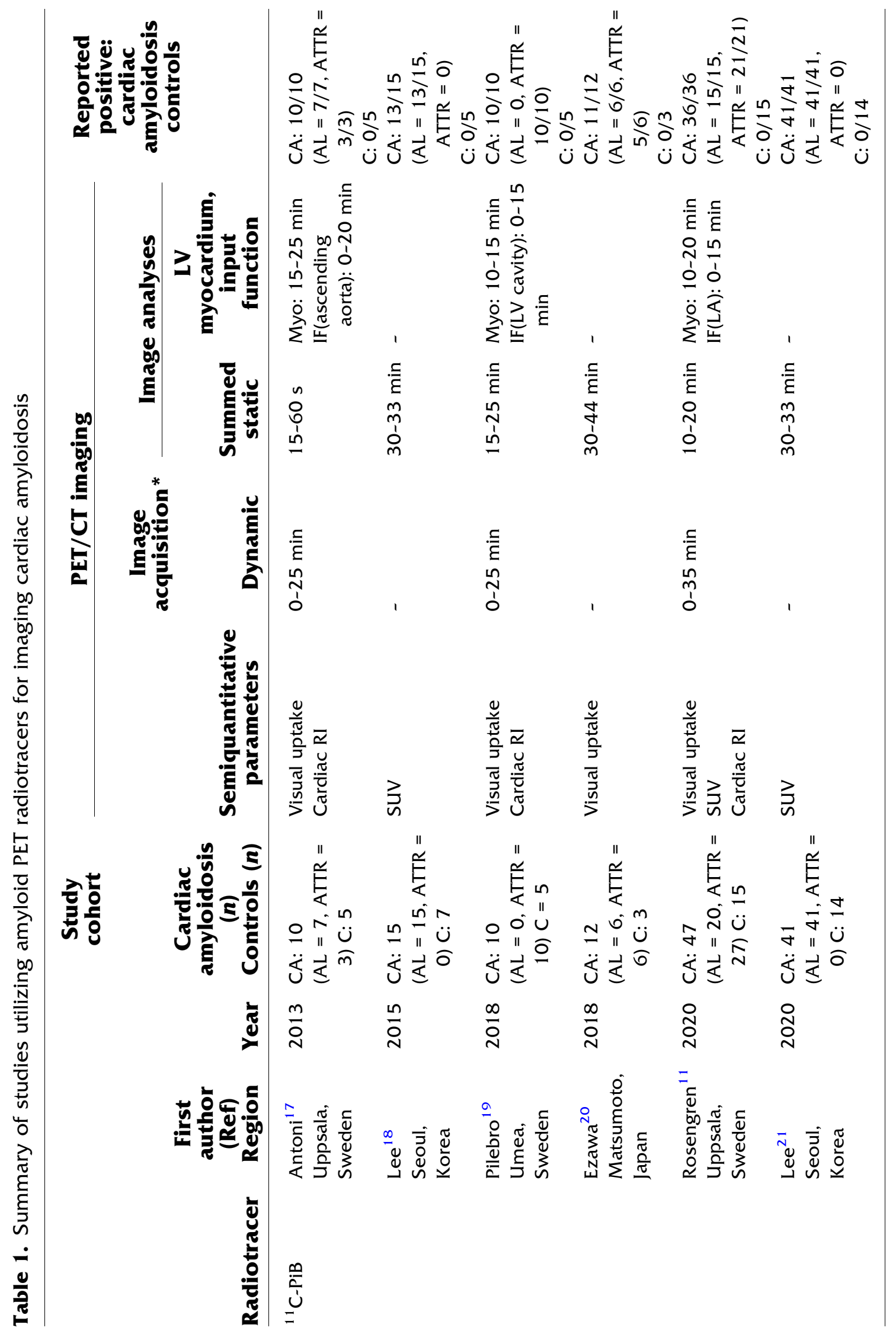




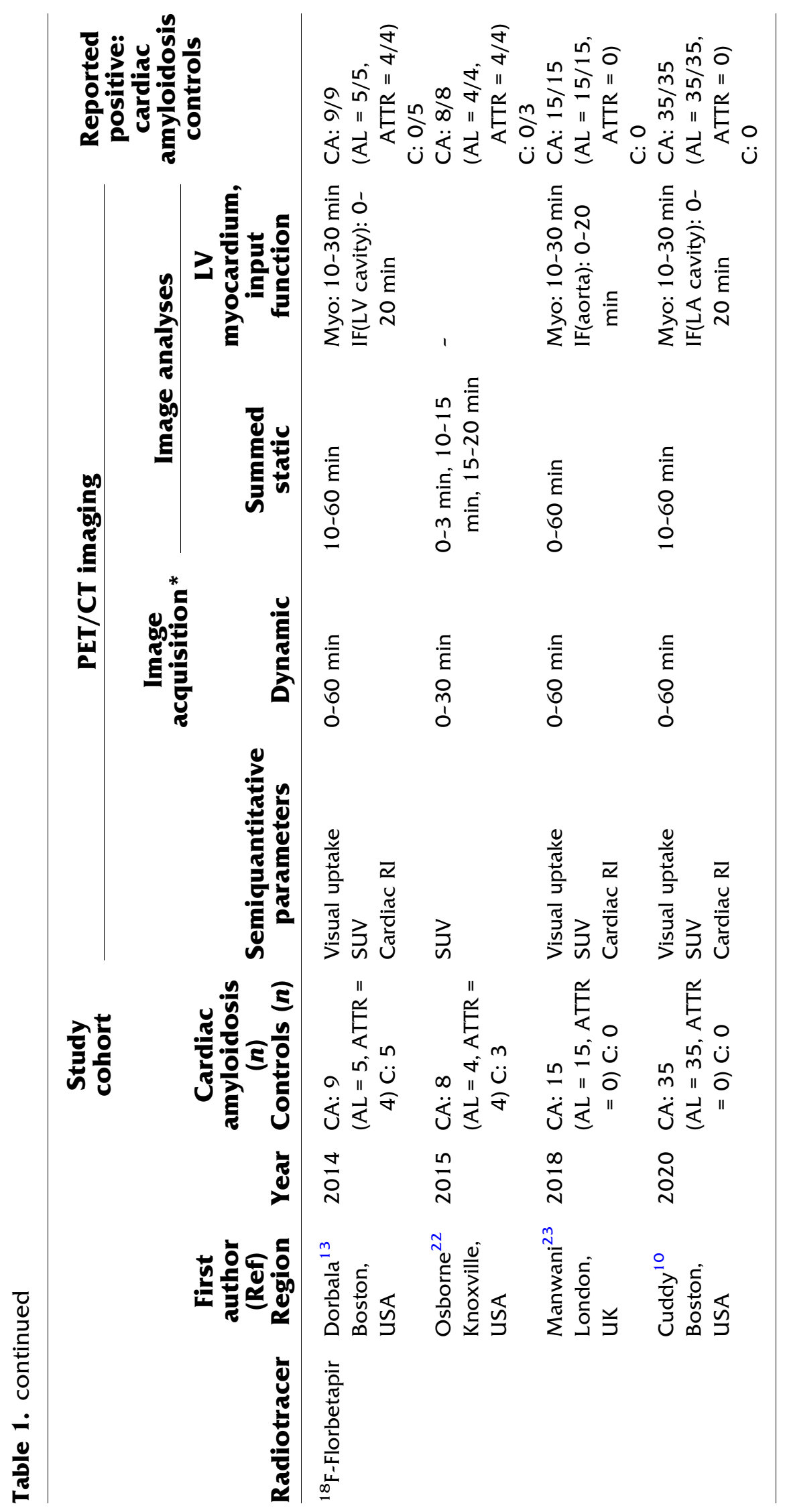




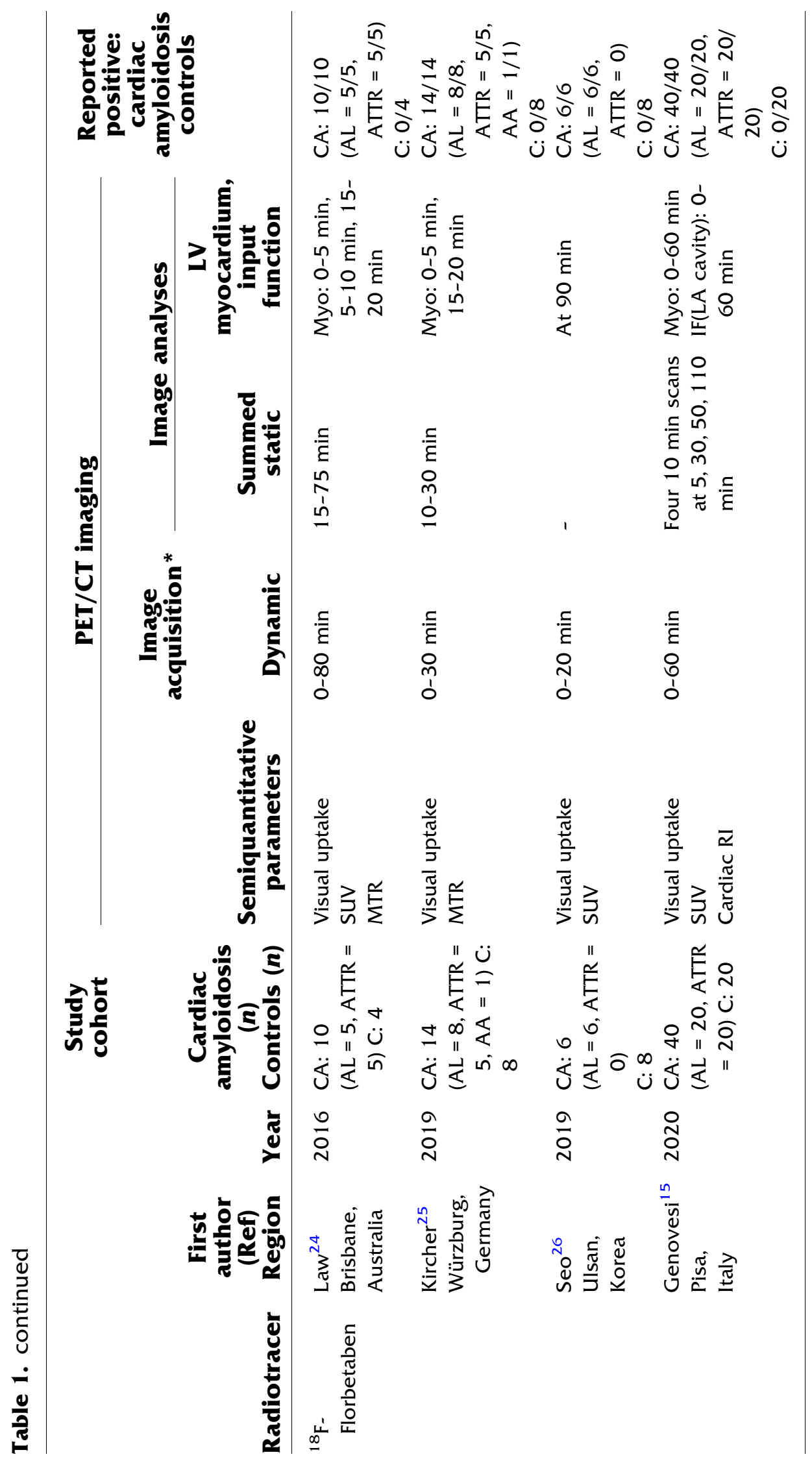




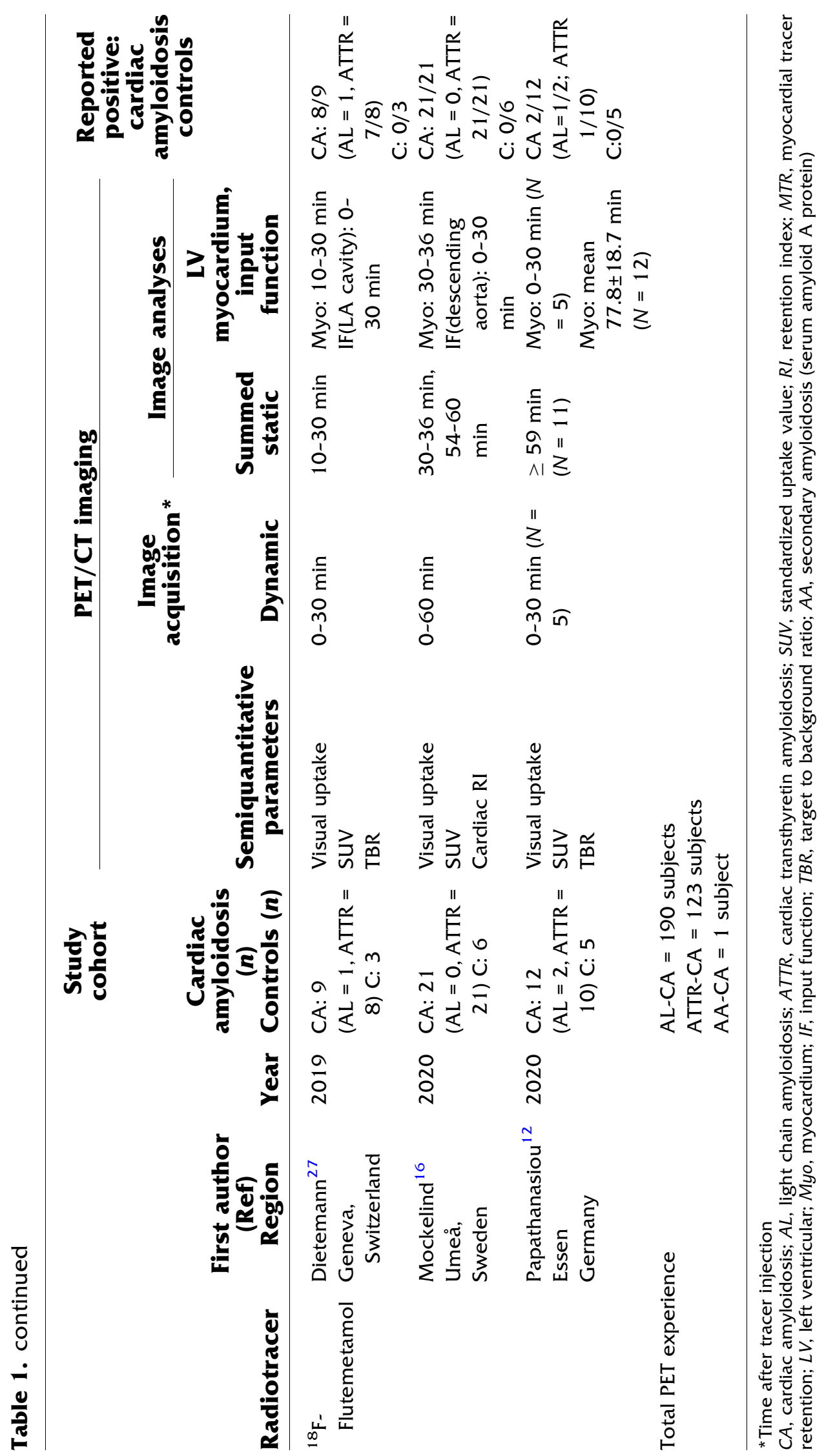


flutemetamol in cardiac amyloidosis ought to avoid delayed imaging. When developing protocols for a novel tracer whose cardiac tracer kinetics are not well defined, a dynamic image acquisition over a wide range of time periods, in this case first 60 minutes, may be helpful to determine the optimal imaging time. Large multicenter studies using standardized protocols are much needed to establish the diagnostic accuracy of amyloid tracer PET imaging in cardiac amyloidosis. As targeted therapies for ATTR-CM and lifesaving therapies for AL-CM are now available, and antifibril therapies are currently under development, this emerging field needs a specific and quantitative measure of myocardial amyloid burden. Targeted amyloid-binding PET tracer imaging has an important role to play and the timing is now!

\section{Disclosures}

Vasvi Singh: Research grant: American Society of Nuclear Cardiology and Pfizer. Sharmila Dorbala: Consulting fees: Pfizer and GE Health Care; Research grants: Pfizer and GE Health Care.

\section{References}

1. Singh V, Falk R, Di Carli MF, Kijewski M, Rapezzi C, Dorbala S State-of-the-art radionuclide imaging in cardiac transthyretin amyloidosis. J Nucl Cardiol 2019;26:158-73.

2. Dorbala S, Cuddy S, Falk RH. How to image cardiac amyloidosis: A practical approach. JACC Cardiovasc Imaging 2020;13:136883.

3. Phelan D, Collier P, Thavendiranathan P, Popovic ZB, Hanna M, Plana JC, et al. Relative apical sparing of longitudinal strain using two-dimensional speckle-tracking echocardiography is both sensitive and specific for the diagnosis of cardiac amyloidosis. Heart 2012;98:1442-8.

4. Singh V, Soman P, Malhotra S. Reduced diagnostic accuracy of apical-sparing strain abnormality for cardiac amyloidosis in patients with chronic kidney disease. J Am Soc Echocardiogr 2020;33:913-6.

5. Maurer MS, Schwartz JH, Gundapaneni B, Elliott PM, Merlini G, Waddington-Cruz $\mathrm{M}$, et al. Tafamidis treatment for patients with transthyretin amyloid cardiomyopathy. N Engl J Med 2018;379:1007-16.

6. Alexander KM, Singh A, Falk RH. Novel pharmacotherapies for cardiac amyloidosis. Pharmacol Ther 2017;180:129-38.

7. Gillmore JD, Maurer MS, Falk RH, Merlini G, Damy T, Dispenzieri A, et al. Nonbiopsy diagnosis of cardiac transthyretin amyloidosis. Circulation 2016;133:2404-12.

8. Dorbala S, Kijewski MF, Park MA. Quantitative bone-avid tracer SPECT/CT for cardiac amyloidosis: A crucial step forward. JACC Cardiovasc Imaging 2020;13:1364-7.

9. Kim YJ, Ha S, Kim YI. Cardiac amyloidosis imaging with amyloid positron emission tomography: A systematic review and meta-analysis. J Nucl Cardiol 2020;27:123-32.
10. Cuddy SAM, Bravo PE, Falk RH, El-Sady S, Kijewski MF, Park $\mathrm{MA}$, et al. Improved quantification of cardiac amyloid burden in systemic light chain amyloidosis: Redefining early disease? JACC Cardiovasc Imaging 2020;13:1325-36.

11. Rosengren S, Skibsted Clemmensen T, Tolbod L, Granstam SO, Eiskjaer H, Wikstrom G, et al. Diagnostic accuracy of [(11)C]PIB positron emission tomography for detection of cardiac amyloidosis. JACC Cardiovasc Imaging 2020;13:1337-47.

12. Papathanasiou M, Kessler L, Carpinteiro A, Hagenacker T, Nensa F, Umutlu L, et al. (18)F-flutemetamol positron emission tomography in cardiac amyloidosis. J Nucl Cardiol 2020. https://doi.org/ 10.1007/s12350-020-02363-2.

13. Dorbala S, Vangala D, Semer J, Strader C, Bruyere JR Jr, Di Carli $\mathrm{MF}$, et al. Imaging cardiac amyloidosis: A pilot study using (18)Fflorbetapir positron emission tomography. Eur J Nucl Med Mol Imaging 2014;41:1652-62.

14. Park MA, Padera RF, Belanger A, Dubey S, Hwang DH, Veeranna $\mathrm{V}$, et al. 18F-florbetapir binds specifically to myocardial light chain and transthyretin amyloid deposits: Autoradiography study. Circ Cardiovasc Imaging 2015;8:e002954.

15. Genovesi D, Vergaro G, Giorgetti A, Marzullo P, Scipioni M, Santarelli MF, et al. [18F]-florbetaben PET/CT for differential diagnosis among cardiac immunoglobulin light chain, transthyretin amyloidosis, and mimicking conditions. JACC Cardiovasc Imaging 2020. https://doi.org/10.1016/j.jcmg.2020.05.031.

16. Mockelind S, Axelsson J, Pilebro B, Lindqvist P, Suhr OB, Sundstrom T. Quantification of cardiac amyloid with [(18)F]Flutemetamol in patients with V30M hereditary transthyretin amyloidosis. Amyloid 2020;27:191-9.

17. Antoni G, Lubberink M, Estrada S, Axelsson J, Carlson K, Lindsjo $\mathrm{L}$, et al. In vivo visualization of amyloid deposits in the heart with 11C-PIB and PET. J Nucl Med 2013;54:213-20.

18. Lee SP, Lee ES, Choi H, Im HJ, Koh Y, Lee MH, et al. 11CPittsburgh B PET imaging in cardiac amyloidosis. JACC Cardiovasc Imaging 2015;8:50-9.

19. Pilebro B, Arvidsson S, Lindqvist P, Sundstrom T, Westermark P, Antoni G, et al. Positron emission tomography (PET) utilizing Pittsburgh compound B (PIB) for detection of amyloid heart deposits in hereditary transthyretin amyloidosis (ATTR). J Nucl Cardiol 2018;25:240-8.

20. Ezawa N, Katoh N, Oguchi K, Yoshinaga T, Yazaki M, Sekijima Y. Visualization of multiple organ amyloid involvement in systemic amyloidosis using (11)C-PiB PET imaging. Eur J Nucl Med Mol Imaging 2018;45:452-61.

21. Lee SP, Suh HY, Park S, Oh S, Kwak SG, Kim HM, et al. Pittsburgh B compound positron emission tomography in patients with AL cardiac amyloidosis. J Am Coll Cardiol 2020;75:380-90.

22. Osborne DR, Acuff SN, Stuckey A, Wall JS. A routine PET/CT protocol with streamlined calculations for assessing cardiac amyloidosis using (18)F-Florbetapir. Front Cardiovasc Med 2015;2:23.

23. Manwani R, Page J, Lane T, Burniston M, Skillen A, Lachmann $\mathrm{HJ}$, et al. A pilot study demonstrating cardiac uptake with $18 \mathrm{~F}-$ florbetapir PET in AL amyloidosis patients with cardiac involvement. Amyloid: Int J Exp Clin Investig 2018;25:247-52.

24. Law WP, Wang WY, Moore PT, Mollee PN, Ng AC. Cardiac amyloid imaging with 18F-Florbetaben PET: A pilot study. J Nucl Med 2016;57:1733-9.

25. Kircher M, Ihne S, Brumberg J, Morbach C, Knop S, Kortum KM, et al. Detection of cardiac amyloidosis with (18)F-Florbetaben$\mathrm{PET} / \mathrm{CT}$ in comparison to echocardiography, cardiac MRI and 
DPD-scintigraphy. Eur J Nucl Med Mol Imaging 2019;46:140716.

26. Seo M, Cha HJ, Kim M, Park SH, Lim JH, Choi Y, et al. Clinical utility of 18F-Florbetaben PET for detecting amyloidosis associated with multiple myeloma: A prospective case-control study. Clin Nucl Med 2019;44:e503-9.
27. Dietemann S, Nkoulou R. Amyloid PET imaging in cardiac amyloidosis: A pilot study using (18)F-flutemetamol positron emission tomography. Ann Nucl Med 2019;33:624-8.

Publisher's Note Springer Nature remains neutral with regard to jurisdictional claims in published maps and institutional affiliations. 